\title{
Nutritional Composition and $\alpha$-Glucosidase Inhibitory Activity of Five Chinese Vinegars
}

\author{
JunFeng FAN ${ }^{1}$, YanYan ZHANG ${ }^{2}$, LinNa ZHOU $^{1}$, ZaiGui LI $^{3 *}$, BoLin ZHANG ${ }^{1}$, \\ Masayoshi SAITO ${ }^{4}$ and XiaoNan WANG ${ }^{1}$ \\ 1 College of Bioscience and Biotechnology, Beijing Forestry University (Haidian, Beijing \\ 100083, China) \\ 2 Department of Food Science, Beijing Agricultural College (Changping, Beijing 102206, China) \\ 3 College of Food Science \& Nutritional Engineering, China Agricultural University (Haidian, \\ Beijing 100083, China) \\ 4 Program director, Japan International Research Center for Agricultural Sciences (Tsukuba, Iba- \\ raki 305-8686, Japan)
}

\begin{abstract}
Shanxi aged vinegar (SAV) and Zhenjiang aromatic vinegars (ZAV) are representative of minor grain crop vinegars in the north of China and rice vinegars in the south, respectively. The nutritional composition of five Chinese vinegars, including three typical SAV and two ZAV, were determined. Investigations showed that these vinegars differed from each other in terms of the organic acid content (75.32-175.95 g/L), and amino acids (1215.9-2878.8 mg/100 g). SAV, especially oat vinegar, were 9-40, 2-211, 4-210, 1-3 and 1-1.5 times higher than ZAV in calcium, phosphorus, iron, zinc, and manganese contents, respectively. Nicotinic acid, nicotinamide, phenolics, alkaloids and saponin in these vinegars were shown to be $2.33-37.83 \mathrm{mg} / 100 \mathrm{~g}, 1.23-35.03$ $\mathrm{mg} / 100 \mathrm{~g}, 382.4-4518.1 \mu \mathrm{g} / \mathrm{mL}, 0.088-1.209 \mathrm{mg} / \mathrm{mL}$, and $0.183-0.662 \mathrm{mg} / \mathrm{mL}$, respectively. SAV seemed relatively better than ZAV in terms of essential amino acids, minerals and vitamins and also exhibited strong $\alpha$-glucosidase inhibitory activities. These results suggested that both types of typical Chinese vinegars could be used as health foods, despite their different raw materials and processing technologies.
\end{abstract}

Discipline: Food

Additional key words: minor grain crop, organic acid, amino acid

\section{Introduction}

Vinegar has been consumed as a food condiment and preservative in the Chinese diet for more than 3,000 years. There are currently at least 14 main types on the market for different raw material and processing technologies, including Zhenjiang aromatic vinegar (ZAV), Shanxi aged vinegar (SAV), Jiangzhe rose vinegar $(\mathrm{JRV})$, and Baoning bran vinegar $(\mathrm{BBV})^{20}$. Among these, ZAV and SAV are the most famous and mainly popular in the south and north of China, respectively.

ZAV generally employs rice and rice bran as major raw materials, whereas SAV uses minor grain crops (sorghum, oat, and tartary buckwheat) as the main raw materials with a very large dosage of great koji (composed of wheat bran and pea, and comprising around half the raw materials). These minor grain crops are traditionally treasured as health foods for their special nutritive value and functional activity. The manufacturing flowcharts for both types of vinegars are shown in Fig. 1 . With respect to processing procedures, ZAV employ liquid-state fermentation in saccharification and alcohol fermentation, and solid-state fermentation in acetic acid fermentation respectively, while the entire SAV process takes place in the solid state. In particular, ZAV has only an aging process of less than three months without a roasting process following acetic acid fermentation, while SAV has a 6-day fuming (roasting) period and at least 18 months of aging. These processes endow SAV with increased concentrations of organic acids, flavor and nutrients due to chemical and enzymatic reactions ${ }^{20}$, which explains the great variation in quality and nutri- 
Raw material

Koji preparation $\rightarrow$ Koji $\rightarrow \downarrow$

Saccharification

Yeast culture $\rightarrow \downarrow$

Alcohol fermentation

Acetic-acid bacteria $\rightarrow \downarrow \leftarrow$ Rice hull and wheat bran

Acetic acid fermentation (solid)

\author{
$\swarrow \quad \searrow$
}

Roasting $\left(80^{\circ} \mathrm{C}\right.$ for 6 days $)$

$\downarrow$

Maturation (18 months)

$\downarrow$

Shanxi Aged Vinegar (SAV)

Fig. 1. Flowcharts for manufacturing SAV and ZAV used in the present study
Maturation (less than 3 months)

$\downarrow$

Zhenjiang Aromatic Vinegar (ZAV) ents among both types of vinegars.

Besides as an acid seasoning, vinegar has also been used for medicinal purposes in traditional Chinese medi$\operatorname{cine}^{30}$. Recently, the health benefits of vinegar have attracted much attention. Studies have shown that vinegar possesses a wide spectrum of physiological effects, including regulating blood pressure ${ }^{19}$, assisting calcium absorption $^{18}$, improving blood fluidity ${ }^{31}$, antioxidant activities $^{26}$, and antithrombotic and fibrinolytic activity ${ }^{11}$. A steady rise in consumer demand has seen an increase from 2.5 million tons in 2004 to 3.4 million tons in 2009. However, information concerning the detailed chemical composition of these Chinese vinegars remains extremely limited, despite such widespread consumption of SAV or ZAV.

Diabetes mellitus has become a common disease in recent years. An $\alpha$-Glucosidase inhibitor is usually used to prevent or medically treat typeII diabetes by combining with intestinal $\alpha$-glucosidase and blocking the uptake of postprandial blood glucose. A recent study has also demonstrated that vinegar can significantly regulate postprandial hyperglycemia ${ }^{10}$. The mechanism by which 
Table 1. The raw materials, koji, major manufacture processing of vinegars

\begin{tabular}{|c|c|c|c|}
\hline & SV & ZHV & LRV \\
\hline \multirow[t]{6}{*}{ Materials $(\%, \mathrm{w} / \mathrm{v})$} & Sorghum $(45 \%)$ & Rice $(30 \%)$ & Rice $(15 \%)$ \\
\hline & Wheat bran (14\%) & Wheat bran $(40 \%)$ & Starch $(24 \%)$ \\
\hline & Rice hull (14\%) & Rice hull (28\%) & Wheat bran $(15 \%)$ \\
\hline & Great koji (27\%, barley, & Wheat koji $(2 \%)$ & Rice hull (15\%) \\
\hline & oats and peas) & & Wheat koji $(6 \%)$ \\
\hline & & & Yeast powder $(3 \%)$ \\
\hline Microorganisms in koji & Aspergillus & Rhizopus & Rhizopus, yeast \\
\hline Saccharification & Solid state & Liquid state & Liquid state \\
\hline Alcohol fermentation & Solid state & Liquid state & Liquid state \\
\hline Acetic acid fermentation & Solid state & Solid state & Solid state \\
\hline Roasting & Yes & No & No \\
\hline Aging & 18 months & 3 months & 2 months \\
\hline Production period (days) & 540 & 90 & 60 \\
\hline
\end{tabular}

\section{Materials and methods}

\section{Materials}

Three typical SAV, including sorghum vinegar $(\mathrm{SV})$, oat vinegar (OV), and tartary buckwheat vinegar (TBV), were obtained from Shanxi Ziyauan Microorganism R \& D Co., Ltd. (Shanxi, China). Two typical ZAV, including Zhenjiang Hengshun vinegar (ZHV) and Liubiju rice vinegar (LRV) were purchased from a local market. The raw materials, koji and major manufacture processing, are shown in Table 1. The SAV use minor grain crops as the main raw material with a large dosage of great koji (27\% of raw materials). Their production takes 18 months. The ZAV, ZHV and LRV employ rice as the main raw material with a small dosage of koji (less than 3\%) and with aging periods of less than three months. In particular, besides rice, LRV also uses starch as a fermentation substrate to cut costs, and adds yeast powder to rice to partly replace koji and accelerate fermentation. The change ratios of the main raw material to vinegar product are 1:3 for SAV, 1:4 for ZHV, and 1:6 for LRV. Only salt is added to these vinegars to avoid over-fermentation after acetic acid fermentation.

Intestinal acetone powders of rat, 4-nitrophenyl $\alpha$ D-glucopyranoside (4-NPG), Folin-Ciocalteu (FC) reagent, and gallic acid were purchased from Sigma Chemical Co. (St. Louis, MO, USA). The other reagents were of analytical grade.

\section{Proximate composition}

Moisture and ash were estimated using the standard $\mathrm{AOAC}^{2}$ methods. Protein content was calculated from the nitrogen content $(\% \mathrm{~N} \times 6.25)$, as analyzed by the Kjeldahl method. The carbohydrate content was examined using the phenol-sulfuric acid colorimetric method $^{9}$. The lipid content was determined using a Soxhlet apparatus according to the procedure described by Huang $^{16}$ and the $\mathrm{pH}$ was measured by a digital $\mathrm{pH}$ meter. Titratable acidity was determined as acetic acid per $100 \mathrm{~mL}$ using the standard method ${ }^{1}$.

\section{Organic acids}

Before analysis, a $6 \mathrm{~mL}$ aliquot of vinegar was diluted to $20 \mathrm{~mL}$ with water. To eliminate interference, 5 $\mathrm{mL}$ of this solution were passed through a C18 SPE cartridge, which had been previously activated with 2 $\mathrm{mL}$ of a 9:1 (v/v) $\mathrm{H}_{2} \mathrm{O}: \mathrm{CH}_{3} \mathrm{OH}$ mixture. The cartridge was then washed with $2 \mathrm{~mL}$ of water and the collected eluates were diluted to $25 \mathrm{~mL}$. A $10 \mu \mathrm{L}$ aliquot of this solution was loaded onto a Shimadzu HPLC system to analyze the organic acids (acetic, citric, tartaric, gluconic, malic, succinic, and lactic acids). The HPLC system was equipped with a high pressure pump $(0.6 \mathrm{~mL} /$ min), at $45^{\circ} \mathrm{C}$, using an ionic exchange Aminex HPX$87 \mathrm{H}$ column $(300 \times 7.8 \mathrm{~mm}, 9 \mu \mathrm{m})$ from Bio-Rad and a diode array detector $(210 \mathrm{~nm})$ was used for the organic acid. The mobile phase was a $0.005 \mathrm{~mol} / \mathrm{L} \mathrm{H}_{2} \mathrm{SO}_{4}$ solution $(\mathrm{pH} 2.1)$. 


\section{Amino acids}

For the analysis of amino acid in vinegar, thirty milliliters of vinegar was filtered and concentrated under reduced pressure into a gummy residue, $1 \mathrm{~mL}$ of which was mixed with $3 \mathrm{~mL}$ of $6 \mathrm{~N} \mathrm{HCl}$ and hydrolyzed at $110^{\circ} \mathrm{C}$ for $24 \mathrm{~h}$. Subsequently, the hydrolysate was filtered and an aliquot of $0.5 \mathrm{~mL}$ was dried under a vacuum. Three milliliters of the citrate buffer ( $\mathrm{pH} 2.2)$ were added to reconstitute the sample and $10 \mu \mathrm{L}$ was used in loading into the amino acid analyzer (S433, SYKAM Co. Ltd., Munich, Germany).

\section{Minerals}

Calcium, phosphorus, iron, zinc, iodine and manganese were determined using an atomic absorption spectrophotometer (Spectra-AA220, Varian Co., Palo Alto, CA, USA) after digestion in mixed acids (nitric acid : perchloric acid $=4: 1$ ). The selenium content was determined using the fluorometric method of Huang ${ }^{16}$.

\section{Vitamin B complexes}

Thiamine and riboflavin were analyzed using a Fluorometer (RF5300PC, Shimazu, Kyoto, Japan) and niacin (nicotinic acid and nicotinamide) was analyzed by a colorimetric method using a spectrophotometer (UV mini-1240, Shimazu, Japan) by following the standard methods of the AOAC ${ }^{2}$.

\section{Total phenolics}

A sephadex C-18 column was used to separate the phenols from these compounds. Briefly, $0.2 \mathrm{~mL}$ of vinegar was passed through the column, which was then washed 3 times with $2 \mathrm{~mL}$ of water and the adsorbed phenols were eluted 3 times with $3 \mathrm{~mL}$ of methanol on the basis of UV monitoring. At the end of the separation, the methanolic fractions were incorporated and dried and the total phenolic content in the extract was measured spectrophotometrically using the FolinCiocalteu method ${ }^{24}$.

\section{Total alkaloid extraction and analysis}

Vinegar $(30 \mathrm{~mL})$ was filtered and concentrated under reduced pressure into a gummy residue, which was extracted twice with $\mathrm{HCl}(1 \mathrm{~N}, 25 \mathrm{~mL})$ by refluxing on a water bath for $30 \mathrm{~min}$. After cooling, the solution was filtered into a volumetric flask $(250 \mathrm{~mL})$. Alkaloids were liberated at $\mathrm{pH} 9.8$ from the filtrate by adding an aqueous solution of $0.7 \mathrm{M}$ sodium carbonate and extracted with methylene chloride $(3 \times 30 \mathrm{~mL})$ in a separator funnel. The latter extract was dried over anhydrous sodium sulfate to yield the total alkaloid, which was quantified by bromophenol blue colorimetry ${ }^{21}$ using ber- berine hydrochloride as standard.

\section{Total saponin extraction and analysis}

Extraction and estimation of total saponin were accomplished as described by $\mathrm{He}$ et al. ${ }^{14}$

\section{0. $\alpha$-Glucosidase inhibiting activity}

The inhibitory activity of vinegar against rat $\alpha$ glucosidase was determined by measuring the formation of 4-nitrophenol by $\alpha$-glucosidase following the reaction with 4-nitrophenyl $\alpha$-D-glucopyranoside (4-PNP) as described by Yamaki and Mori ${ }^{32}$. The inhibitory activity of vinegar was measured according to the protocol of a micro-well kit $(0.4 \mathrm{ml} \times 96$ flat-bottom wells, Sumitomo Bakelite Co., Ltd., Tokyo, Japan). Vinegar was neutralized to $\mathrm{pH} 7.0$ by using $2 \mathrm{~N} \mathrm{NaOH}$ to eliminate the effect of the acetic acid on $\alpha$-glucosidase. A serial twofold dilution of the neutralized vinegar in microtiter flat-bottom 96-well plates were mixed with $50 \mu \mathrm{L} \alpha-$ glucosidase $(25 \mathrm{mg} / \mathrm{mL}), 50 \mu \mathrm{L} 4-\mathrm{NPG}(0.9133 \mathrm{mg} / \mathrm{mL})$ and $120 \mu \mathrm{L} 0.5 \mathrm{M}$ phosphate buffer ( $\mathrm{pH}$ 6.7). The mixtures were then incubated at $37^{\circ} \mathrm{C}$ for $60 \mathrm{~min}$ and sodium carbonate $(50 \mu \mathrm{L}, 0.67 \mathrm{M})$ was added to stop the reaction. The absorbance of the reactants was measured at $405 \mathrm{~nm}$ using a microplate reader (Model 550, BIORAD Lab., Tokyo, Japan). The $\alpha$-glucosidase inhibitory activity of vinegar was then computed as the slope value from the curve of absorbance versus the vinegar concentration. The higher the slope value, the stronger the anti- $\alpha$-glucosidase activity of the vinegar.

\section{Statistical analysis}

Each experiment was performed in triplicate, with the results expressed as a mean \pm SD. Statistical comparisons were made via one-way analysis of variance, followed by a Duncan multiple-comparison test. Differences were considered significant when the $p$ values were 0.05 .

\section{Results and discussion}

\section{Proximate composition}

The results of the proximate chemical analysis of the five vinegars are shown in Table 2. The moisture contents of these vinegars ranged from $67.53 \%$ in OV to $88.10 \%$ in LRV, and SAV showed lower moisture content than $\mathrm{ZAV}$, which could be related to its longer aging period. The variations in this parameter implied proportional increases or decreases in all nutrients on a wet weight basis. Crude protein contents varied from $1.7 \%$ in LRV to $10.47 \%$ in TBV, and all the SAV were significantly higher than ZAV in this parameter $(p<$ 
Table 2. Chemical components of vinegar $(\%, w / v){ }^{A, B}$

\begin{tabular}{lccccc}
\hline \hline Component & \multicolumn{1}{c}{ SV } & OV & TBV & ZHV & LRV \\
\hline Moisture & $68.10 \pm 2.85^{\mathrm{a}}$ & $67.53 \pm 0.26^{\mathrm{a}}$ & $83.24 \pm 0.77^{\mathrm{b}}$ & $86.74 \pm 0.02^{\mathrm{c}}$ & $88.10 \pm 0.27^{\mathrm{c}}$ \\
Crude protein & $3.86 \pm 0.01^{\mathrm{a}}$ & $6.48 \pm 0.08^{\mathrm{b}}$ & $10.47 \pm 0.09^{\mathrm{c}}$ & $2.27 \pm 0.11^{\mathrm{d}}$ & $1.70 \pm 0.02^{\mathrm{e}}$ \\
Carbohydrate & $0.76 \pm 0.09^{\mathrm{a}}$ & $0.59 \pm 0.007^{\mathrm{b}}$ & $0.33 \pm 0.03^{\mathrm{c}}$ & $0.27 \pm 0.02^{\mathrm{c}}$ & $0.13 \pm 0.005^{\mathrm{d}}$ \\
Total lipid & $0.24 \pm 0.07$ & $0.31 \pm 0.05$ & $0.16 \pm 0.01$ & $0.14 \pm 0.01$ & $0.15 \pm 0.00$ \\
Ash & $4.57 \pm 0.68^{\mathrm{a}}$ & $4.93 \pm 0.20^{\mathrm{a}}$ & $2.58 \pm 0.15^{\mathrm{b}}$ & $3.16 \pm 0.12^{\mathrm{c}}$ & $2.41 \pm 0.17^{\mathrm{b}}$ \\
$\mathrm{pH}$ & $3.60 \pm 0.44$ & $3.71 \pm 0.29$ & $3.68 \pm 0.16$ & $3.38 \pm 0.18$ & $3.59 \pm 0.25$ \\
Titratable acidity & $6.01 \pm 0.02^{\mathrm{c}}$ & $7.78 \pm 0.04^{\mathrm{a}}$ & $6.77 \pm 0.02^{\mathrm{b}}$ & $6.75 \pm 0.04^{\mathrm{b}}$ & $5.27 \pm 0.02^{\mathrm{d}}$ \\
\hline A. Each value is expressed as a mean \pm SD $(\mathrm{n}=3) ;$ Values in a row with different superscripts differ significantly $(\mathrm{p}<$ \\
0.05). \\
в: SV, OV, TBV, ZHV and LRV represent sorghum vinegar, oat vinegar, tartary buckwheat vinegar, Zhenjiang Hengshun \\
vinegar and Liubiju rice vinegar, respectively.
\end{tabular}

Table 3. Organic acid contents (g/L) of vinegars ${ }^{\mathrm{A}, \mathrm{B}}$

\begin{tabular}{lcrrrr}
\hline \hline Organic acid & \multicolumn{1}{c}{ SV } & \multicolumn{1}{c}{ OV } & \multicolumn{1}{c}{ TBV } & \multicolumn{1}{c}{ ZHV } & LRV \\
\hline Tartaric acid & $1.54 \pm 0.16$ & $7.32 \pm 1.15$ & $3.45 \pm 0.21$ & $1.92 \pm 1.11$ & $3.04 \pm 0.09$ \\
Formic acid & $1.80 \pm 0.21$ & $4.51 \pm 0.88$ & $2.90 \pm 0.14$ & $0.3 \pm 0.21$ & $0.27 \pm 0.16$ \\
Malic acid & $4.50 \pm 0$ & $8.57 \pm 0.45$ & $6.50 \pm 0.71$ & $1.92 \pm 1.01$ & $1.09 \pm 0.06$ \\
Lactic acid & $25.50 \pm 4.24$ & $54.38 \pm 0.88$ & $22.50 \pm 2.12$ & $9.38 \pm 3.71$ & $33.04 \pm 11.67$ \\
Acetic acid & $57.05 \pm 1.06$ & $98.13 \pm 2.65$ & $73.5 \pm 7.78$ & $59.25 \pm 26.52$ & $62.63 \pm 6.89$ \\
Citric acid & $0.33 \pm 0.08$ & $1.05 \pm 0.06$ & $1.25 \pm 0.21$ & $1.43 \pm 0.74$ & $2.29 \pm 0.16$ \\
Succinic acid & $0.41 \pm 0.14$ & $0.63 \pm 0.25$ & $0.23 \pm 0.02$ & $0.69 \pm 0.30$ & $0.67 \pm 0.11$ \\
Total & $91.1 \pm 1.03$ & $175.95 \pm 7.73$ & $111.43 \pm 6.05$ & $75.32 \pm 2.71$ & $103.39 \pm 3.49$ \\
\hline
\end{tabular}

A: Each value is expressed as a mean $\pm \operatorname{SD}(n=3)$.

B. SV, OV, TBV, ZHV and LRV represent sorghum vinegar, oat vinegar, tartary buckwheat vinegar, Zhenjiang Hengshun vinegar and Liubiju rice vinegar, respectively.

0.05), while the carbohydrate contents were low for all vinegars. They followed the order: SV $(0.76 \%)>\mathrm{OV}$ $(0.59 \%)>\operatorname{TBV}(0.33 \%)$ and $\mathrm{ZHV}(0.27 \%)>\mathrm{LRV}$ $(0.13 \%)$. The saccharides in these vinegars were also much lower than those in some Japanese vinegars, ranging from 1 to $5 \%{ }^{19}$. Fermentation was generally more thoroughly conducted in Chinese vinegar in order to obtain the considerably sour taste. The five vinegars had lower lipid contents ranging from 0.14 to $0.31 \%$, with no significant difference emerging. The ash contents ranged from 2.41 to $4.93 \%$, with $\mathrm{OV}$ and SV having higher ash contents than the others. Titratable acidity is an important index of vinegar and must exceed $3.5 \%$ (w /v) in accordance with the China State Standard. The vinegars had far higher titratable acidities (5.27-7.78\%).

\section{Organic acids}

The contents of organic acids in the vinegars ana- lyzed are presented in Table 3, with totals ranging from 75.32 to $175.95 \mathrm{~g} / \mathrm{L}$. OV and TBV contained more than the others, while ZHV contained less.

Organic acids are important in imparting vinegar with a suitable taste and flavor. Recent studies showed that organic acids also have important physiological activities, including antihypertensive ${ }^{19}$ and antihyperglycemic effects ${ }^{35}$. The concentrations of acetic acid in the vinegars ranged from $5.7 \%(\mathrm{w} / \mathrm{v})$ to $9.8 \%(\mathrm{w} / \mathrm{v})($ Table 3). According to Cheng et al. ${ }^{7}$, when food containing $10-150 \mathrm{mmol} / \mathrm{L}$ of acetic acid was eaten, the concentration of acetic acid possibly reached the millimolar level in the small intestine. Such a level can have physiological effects, including disaccharidase and angiotensin-I converting enzyme inhibitory activities in the small intestine $^{22}$. The vinegars contained so much acetic acid that they could still reach the millimolar level in the small intestine, even after dilution 6-11 times. 
Lactic acid was found to be the second dominant organic acid (0.9\%-5.4\%) next to acetic acid in the vinegars. This nonvolatile acid would generally intensify the stimulus of acetic acid and make sourness taste more persistence. Tartaric acid showed an obvious difference in different vinegars and the order was: OV $(7.32 \mathrm{~g} / \mathrm{L})>\mathrm{TBV}(3.45 \mathrm{~g} / \mathrm{L})$ and $\operatorname{LRV}(3.04 \mathrm{~g} / \mathrm{L})>$ ZHV (1.92 g/L) and SV (1.54 g/L). Formic and malic acids were high in SAV (1.80-4.51 and 4.50-8.57 g/L, respectively), which were 6-20 and 2-8 times higher than that in ZAV $(0.27-0.3$ and $1.09-1.92 \mathrm{~g} / \mathrm{L}$, respectively). As regards succinic acid, it was present at low concentration and showed no obvious difference between SAV and ZAV.

Citric acid, which is known to be good for health, ranged from 0.33 to $2.29 \mathrm{~g} / \mathrm{L}$ in the vinegars, with $\mathrm{LRV}$ containing more $(2.29 \mathrm{~g} / \mathrm{L})$ than the others (0.33-1.43 g/ L). This concentration was less than that of Japanese vinegars $(2.73-7.66 \mathrm{mg} / \mathrm{mL})^{34}$.

\section{Amino acids}

Table 4 shows the concentrations of various amino acids in the five vinegars, with their totals in order: SV
$(2878.8 \mathrm{mg} / 100 \mathrm{~g})>\mathrm{ZHV}(2465.1 \mathrm{mg} / 100 \mathrm{~g})>\mathrm{TBV}$ $(2332.3 \mathrm{mg} / 100 \mathrm{~g})>\mathrm{OV}(1532.1 \mathrm{mg} / 100 \mathrm{~g})>\mathrm{LRV}$ (1215.9 mg/100 g).

The total amino acids of several Japanese vinegars including rice vinegar, malt vinegar, apple vinegar and onion vinegar reportedly ranged from $30.6 \mathrm{mg} / 100 \mathrm{~mL}$ to $210 \mathrm{mg} / 100 \mathrm{~mL}^{15}$. Compared with these Japanese vinegars, the Chinese vinegars tested in the present study were more abundant in total amino acids, namely 5.8-94 times more than Japanese vinegars. The formation of amino acids in vinegar depends on the raw materials used, the particular fermentation condition and the action and autolysis of the bacteria involved in the process $^{5}$.

These vinegars are rich in all kinds of amino acids. As shown in Table 4, glutamic acid was the most abundant amino acid in these vinegars, and ranged from $294.9 \mathrm{mg} / 100 \mathrm{~g}$ in LRV to $887.5 \mathrm{mg} / 100 \mathrm{~g}$ in SV. Some amino acids, including asparagine, glycine, alanine, valine, leucine, threonine, serine, isoleucine and lysine dominated and accounted for about 55 to $62 \%$ of all the amino acid. Cystine, methionine, tyrosine, phenylalanine, histidine and arginine in these vinegars were mi-

Table 4. Amino acid contents (mg/100g) of vinegar A, B

\begin{tabular}{lccccc}
\hline \hline Amino acid & SV & OV & TBV & ZHV & LRV \\
\hline Asparagine & $227.2 \pm 10.8$ & $130.1 \pm 11.7$ & $205.6 \pm 4.1$ & $421.8 \pm 18.5$ & $51.2 \pm 5.0$ \\
Threonine & $92.0 \pm 13.1$ & $50.0 \pm 3.8$ & $84.2 \pm 2.3$ & $136.9 \pm 5.2$ & $50.9 \pm 2.6$ \\
Serine & $111.7 \pm 11.6$ & $61.5 \pm 3.4$ & $88.3 \pm 3.8$ & $82.4 \pm 6.3$ & $49.9 \pm 4.5$ \\
Glutamic acid & $887.5 \pm 53.5$ & $462.6 \pm 17.4$ & $695.1 \pm 14.9$ & $659.1 \pm 26.2$ & $294.9 \pm 17.1$ \\
Glycine & $175.0 \pm 24.0$ & $99.6 \pm 7.9$ & $141.6 \pm 3.1$ & $131.1 \pm 18.5$ & $80.8 \pm 13.9$ \\
Alanine & $250.9 \pm 14.4$ & $119.0 \pm 9.4$ & $186.8 \pm 11.5$ & $153.3 \pm 14.1$ & $166.9 \pm 7.7$ \\
Cystine & $29.9 \pm 5.2$ & $20.5 \pm 2.1$ & $22.0 \pm 3.6$ & $52.9 \pm 7.4$ & $9.9 \pm 1.4$ \\
Valine & $215.5 \pm 12.0$ & $107.2 \pm 9.1$ & $170.5 \pm 9.3$ & $118.2 \pm 11.1$ & $105.7 \pm 8.0$ \\
Methionine & $20.4 \pm 2.5$ & $11.1 \pm 1.4$ & $19.9 \pm 2.5$ & $22.1 \pm 3.7$ & $20.8 \pm 3.2$ \\
Isoleucine & $105.9 \pm 10.6$ & $51.0 \pm 3.3$ & $85.9 \pm 5.0$ & $81.0 \pm 11.6$ & $57.2 \pm 4.4$ \\
Leucine & $175.3 \pm 14.0$ & $92.6 \pm 4.4$ & $130.6 \pm 9.1$ & $97.6 \pm 9.2$ & $95.5 \pm 7.5$ \\
Tyrosine & $51.0 \pm 4.5$ & $30.6 \pm 1.7$ & $41.5 \pm 2.8$ & $56.3 \pm 7.9$ & $12.0 \pm 1.8$ \\
Phenylalanine & $69.7 \pm 4.7$ & $53.5 \pm 3.5$ & $57.4 \pm 6.3$ & $66.2 \pm 6.2$ & $18.5 \pm 2.3$ \\
Histidine & $52.3 \pm 3.4$ & $29.9 \pm 2.5$ & $44.7 \pm 4.7$ & $74.5 \pm 7.1$ & $9.9 \pm 1.4$ \\
Lysine & $80.5 \pm 5.9$ & $45.7 \pm 3.7$ & $100.6 \pm 8.5$ & $47.6 \pm 5.9$ & $72.0 \pm 4.6$ \\
Arginine & $50.4 \pm 2.3$ & $36.7 \pm 2.6$ & $49.6 \pm 4.2$ & $84.5 \pm 5.2$ & $23.8 \pm 1.3$ \\
Proline & $293.8 \pm 16.6$ & $130.3 \pm 7.8$ & $207.9 \pm 14.3$ & $179.8 \pm 14.6$ & $96.2 \pm 4.6$ \\
Total & $2878.8 \pm 110.9$ & $1532.1 \pm 25.1$ & $2332.3 \pm 10.3$ & $2465.1 \pm 18.1$ & $1215.9 \pm 12.1$ \\
\hline A Each & & & & &
\end{tabular}

${ }^{A}$ : Each value is expressed as a mean $\pm \mathrm{SD}(\mathrm{n}=3)$.

в : SV, OV, TBV, ZHV and LRV represent sorghum vinegar, oat vinegar, tartary buckwheat vinegar, Zhenjiang Hengshun vinegar and Liubiju rice vinegar, respectively. 
nor amino acids, accounting for about 6 to $13 \%$ of the total amino acid. The amino acids influenced the taste and bio-functionality of the vinegars ${ }^{15}$, 29 , while glutamic acid, asparagine, glycine, alanine and methionine could enhance the flavor of the vinegars thanks to their umami taste.

Figure 2 shows the essential amino acid supply, which indicates the percentage ratio of essential amino acids in vinegar $(100 \mathrm{~g})$ relative to its recommended daily allowance. Significant differences emerged in the essential amino acid supply between both types of vinegars, SAV and ZAV. SAV mainly provided valine, while ZHV could provide more threonine. Obvious differences also emerged in the essential amino acid supply among the three SAV. The total value of the essential amino acid supply of SV was the highest (118.4\%) among these vinegars, followed by TBV (101\%), while OV ranked third (63\%). These data suggest that SV is superior in terms of essential amino acid content than the others. The essential amino acids in SV could be derived from its main raw materials, sorghum, which is rich in essential amino acids ${ }^{27}$.

\section{Minerals}

The mineral contents of these vinegars are summarized in Table 5. The data indicated that calcium, phosphorus, iron, zinc and manganese were the major mineral constituents in these vinegars, while iodine and selenium were also detected in appreciable amounts. OV contained the highest amounts of minerals, and LRV the lowest.

Calcium was the predominant mineral in the five vinegars, ranging from $31.735 \mathrm{mg} / \mathrm{kg}$ in LRV to 1246 $\mathrm{mg} / \mathrm{kg}$ in $\mathrm{OV}$ and its content differed significantly among the five vinegars $(p<0.05)$. It is necessary to

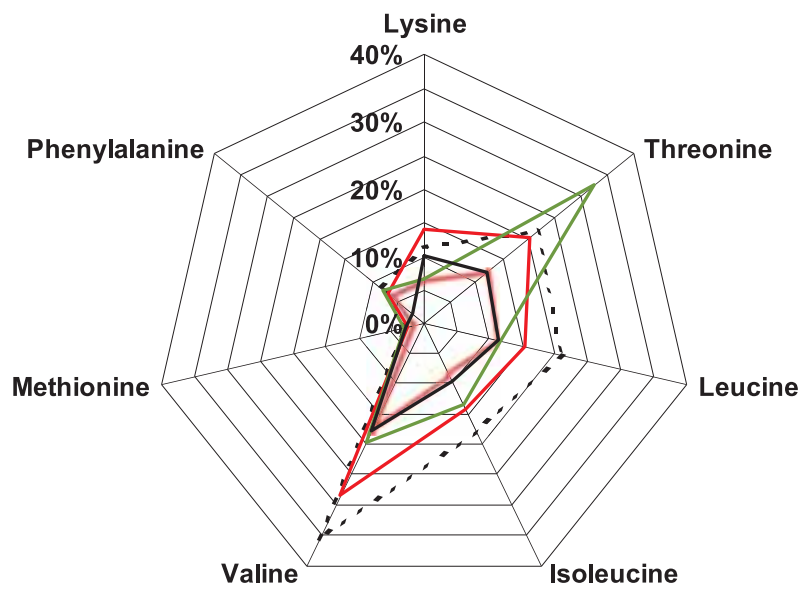

Fig. 2. Percentage ratio of seven of the essential amino acids in vinegar relative to the recommended daily allowance

$\cdots: \mathrm{SV},=$ : OV, — : TBV,

- : ZHV, — : LRV.

emphasize the high calcium content of SAV. All the values were higher in SAV $(651-1246 \mathrm{mg} / \mathrm{kg})$ than those in ZAV (31-134 mg/kg). SV (651 mg/kg), OV (1246 mg/ $\mathrm{kg})$, and TBV $(696 \mathrm{mg} / \mathrm{kg})$, meanwhile, were excellent sources of this mineral, even more so than milk $(\sim 500$ $\mathrm{mg} / \mathrm{kg}$ ). Several clinical studies have shown calcium to be an effective pressure-reducing agent ${ }^{23,36}$. It had been reported that leavening could improve the absorbability of calcium in flour-based products due to the phytate hydrolysis caused by yeast fermentation ${ }^{27}$. Dietary vinegar was also found to enhance intestinal calcium absorption by improving calcium solubility and due to the trophic effect of the acetic acid ${ }^{25}$.

Phosphorus is a component of DNA, RNA, ATP, and also phospholipids, which form all cell membranes,

Table 5. Minerals in vinegar $(\mathrm{mg} / \mathrm{kg}){ }^{\mathrm{A}, \mathrm{B}}$

\begin{tabular}{lccccc}
\hline \hline Mineral & SV & OV & TBV & ZHV & LRV \\
\hline Calcium & $651.370 \pm 49.432^{\mathrm{a}}$ & $1246.365 \pm 71.873^{\mathrm{b}}$ & $695.904 \pm 70.839^{\mathrm{a}}$ & $133.897 \pm 11.468^{\mathrm{c}}$ & $31.735 \pm 6.104^{\mathrm{d}}$ \\
Phosphorus & $3334.577 \pm 124.741^{\mathrm{a}}$ & $4722.563 \pm 390.527^{\mathrm{b}}$ & $3465.652 \pm 221.695^{\mathrm{a}}$ & $1968.038 \pm 77.367^{\mathrm{c}}$ & $22.296 \pm 2.663^{\mathrm{d}}$ \\
Selenium & $0.008 \pm 0.006^{\mathrm{a}}$ & $0.015 \pm 0.009^{\mathrm{a}}$ & $0.014 \pm 0.001^{\mathrm{a}}$ & $0.012 \pm 0.006^{\mathrm{a}}$ & $0.001 \pm 0.003^{\mathrm{a}}$ \\
Iron & $161.991 \pm 22.364^{\mathrm{a}}$ & $304.603 \pm 28.671^{\mathrm{b}}$ & $139.636 \pm 18.479^{\mathrm{a}}$ & $38.254 \pm 4.728^{\mathrm{c}}$ & $1.452 \pm 0.083^{\mathrm{d}}$ \\
Zinc & $22.207 \pm 1.331^{\mathrm{a}}$ & $37.816 \pm 1.918^{\mathrm{b}}$ & $17.704 \pm 2.104^{\mathrm{c}}$ & $16.584 \pm 1.427^{\mathrm{c}}$ & $0.243 \pm 0.016^{\mathrm{d}}$ \\
Manganese & $45.168 \pm 3.295^{\mathrm{a}}$ & $70.369 \pm 5.623^{\mathrm{b}}$ & $39.775 \pm 4.768^{\mathrm{a}}$ & $45.318 \pm 4.442^{\mathrm{a}}$ & $0.038 \pm 0.009^{\mathrm{c}}$ \\
Iodine & $0.173 \pm 0.011^{\mathrm{a}}$ & $0.528 \pm 0.107^{\mathrm{b}}$ & $0.098 \pm 0.028^{\mathrm{c}}$ & $0.314 \pm 0.027^{\mathrm{d}}$ & $0.066 \pm 0.039^{\mathrm{c}}$ \\
\hline
\end{tabular}

A :Each value is expressed as a mean \pm SD $(n=3)$; Values in a row with different superscripts differ significantly $(p<$ $0.05)$.

в :SV, OV, TBV, ZHV and LRV represent sorghum vinegar, oat vinegar, tartary buckwheat vinegar, Zhenjiang Hengshun vinegar and Liubiju rice vinegar, respectively. 
making it an essential element for all living cells. Phosphorus also turned out to be the predominant mineral in these vinegars. In terms of phosphorus content, SAV was obviously higher than ZAV. The highest value was found in OV $(4722.6 \mathrm{mg} / \mathrm{kg})$, followed by SV (3465.6 $\mathrm{mg} / \mathrm{kg}$ ) and TBV (3334.6 mg/kg), ZAV (1968.0 mg/kg) ranked fourth, and the content of LRV $(22.3 \mathrm{mg} / \mathrm{kg})$ was the lowest.

SAV also seemed to be a richer source of iron $(139.9-304.6 \mathrm{mg} / \mathrm{kg}$ ), while OV had the highest iron content among the vinegars. Figure 3 also shows the mineral supply, the percentage ratio of minerals in vinegar $(100 \mathrm{~g})$ relative to the recommended daily allowance. As shown in Fig. 3, iron was the most noticeable mineral supplied by SAV. The iron supply was from $139.6 \%$ of TBV to $304.6 \%$ of OV. Consuming less than fifty gram of OV could provide sufficient iron to meet the recommended daily allowance of $15 \mathrm{mg}$ per person. Cereals generally contain small amounts of phytate but sufficient to inhibit iron absorption. However, fermentation could cause the degradation of phytate, finally changing cereal with low iron bioavailability into a diet of high iron bioavailability ${ }^{27}$. Furthermore, vinegar and acetic acid could also increase iron absorption ${ }^{25}$. Therefore, the iron in vinegar was speculated as having high human bioavailability.

$\mathrm{OV}$ and SV contained to show relatively high amounts of zinc (37.8 and $22.2 \mathrm{mg} / \mathrm{kg}$, respectively) (Table 5). TBV and ZHV contained almost equivalent amounts of zinc (17.7 and $16.6 \mathrm{mg} / \mathrm{kg}$, respectively),

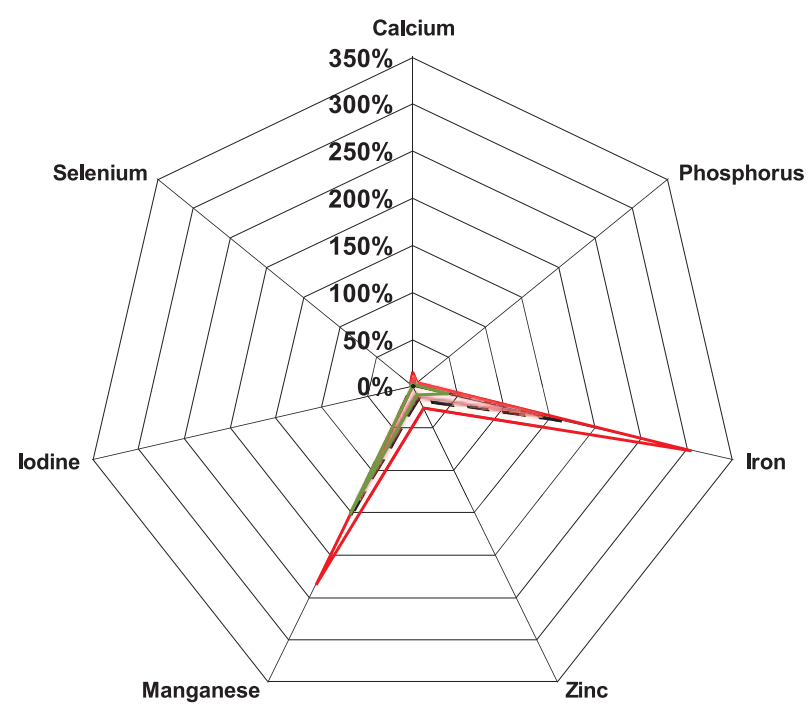

Fig. 3. Percentage ratio of seven of the essential minerals in vinegar relative to the recommended daily allowance $\cdots:$ SV,
- : OV, - : while LRV had the lowest zinc content $(0.243 \mathrm{mg} / \mathrm{kg})$. As recommended by the Food and Nutrition Board, National Research Council ${ }^{12}$, the recommended daily amount is $16 \mathrm{mg}$. In vivo investigations of the effect of phytate on zinc bioavailability showed a similar relationship for iron and phytate, and food fermentation and processing that decreased the phytate content improved zinc absorption ${ }^{27}$.

Vinegar was also a rich source of manganese. The richest source was OV $(70.369 \mathrm{mg} / \mathrm{kg})$, followed by SV (45.168 mg/kg) and ZHV (45.318 mg/kg) (Table 4). The value of TBV ranked fourth $(39.775 \mathrm{mg} / \mathrm{kg})$, and LRV was the lowest $(0.038 \mathrm{mg} / \mathrm{kg})$. The daily manganese requirement is $3-5 \mathrm{mg}$, which can be covered by $50 \mathrm{~g}$ of OV. The manganese supply was $234.6 \%$ for OV, $151.1 \%$ for ZHV, $152.6 \%$ for SV, and $132.6 \%$ for TBV, respectively (Fig. 3). These data show that consuming less than $100 \mathrm{~g}$ of vinegar can meet the entire manganese requirement of the body. The human bioavailability of manganese in vinegars may be compromised by the co-presence of some components such as calcium, iron and phytates that can render manganese unavailable for absorption in the intestine. Therefore, further research must be conducted to elucidate the human bioavailability of manganese in vinegar.

Variations in the contents of minerals for vinegars might be due to their raw materials and manufacturing methods. SAV is mainly made from minor grain crops, such as sorghum, oat, tartary buckwheat, and pea, which tend to be grown in drought and semi-arid areas. Mineral-efficient varieties of plants are more drought resistant and require less irrigation. In addition, SAV generally has a longer roasting and aging process (more than 18 months), which makes it condensed.

\section{Vitamin B complexes}

Table 6 shows the nicotinic acid, nicotinamide, thiamine and riboflavin contents of the five vinegars. According to the results, thiamine and riboflavin were low in SAV and ZHV, and not detected in LBV. However, large amounts of nicotinic acid and nicotinamide were present at contents ranging from 2.33 to $37.83 \mathrm{mg} /$ $100 \mathrm{~g}$ and 1.23 to $35.03 \mathrm{mg} / 100 \mathrm{~g}$, respectively.

The two niacin vitamers, nicotinic acid and nicotinamide, contribute to the coenzymes nicotinamide adenine dinucleotide (NAD) and nicotinamide adenine dinucleotide phosphate (NADP) which are involved in the physiological redox reactions of carbohydrates, fatty acids and amino acid metabolism. Riboflavin acts as a catalyst for the mitochondrial electron transport chain in its coenzymes. Interestingly, SAV was much richer than ZHV in nicotinic acid, nicotinamide and thiamine, mak- 
Table 6. Vitamins, total phenolics, alkaloids and saponin in vinegar A, B

\begin{tabular}{lcrrrc}
\hline \hline & SV & OV & TBV & ZHV & LRV \\
\hline Nicotinic acid $(\mathrm{mg} / 100 \mathrm{~g})$ & $29.24 \pm 2.63^{\mathrm{a}}$ & $37.83 \pm 1.48^{\mathrm{b}}$ & $34.12 \pm 1.53^{\mathrm{a}}$ & $11.91 \pm 0.15^{\mathrm{c}}$ & $2.33 \pm 0.03^{\mathrm{d}}$ \\
Nicotinamide $(\mathrm{mg} / 100 \mathrm{~g})$ & $24.48 \pm 1.24^{\mathrm{a}}$ & $35.03 \pm 2.74^{\mathrm{b}}$ & $13.37 \pm 0.30^{\mathrm{c}}$ & $13.52 \pm 1.28^{\mathrm{c}}$ & $1.23 \pm 0.05^{\mathrm{d}}$ \\
Thiamine $(\mu \mathrm{g} / 100 \mathrm{~g})$ & $0.37 \pm 0.09^{\mathrm{a}}$ & $0.71 \pm 0.06^{\mathrm{b}}$ & $0.46 \pm 0.07^{\mathrm{a}}$ & $0.13 \pm 0.01^{\mathrm{c}}$ & $-^{\mathrm{c}}$ \\
Riboflavin $(\mathrm{mg} / 100 \mathrm{~g})$ & $1.45 \pm 0.05^{\mathrm{a}}$ & $1.63 \pm 0.34^{\mathrm{a}}$ & $0.89 \pm 0.04^{\mathrm{b}}$ & $2.1 \pm 0.05^{\mathrm{c}}$ & $-^{\mathrm{c}}$ \\
Phenolics $(\mu \mathrm{g} / \mathrm{mL})$ & $3964.7 \pm 64.2^{\mathrm{a}}$ & $2812.1 \pm 52.8^{\mathrm{b}}$ & $4518.1 \pm 107.5^{\mathrm{c}}$ & $1564.2 \pm 103.7^{\mathrm{d}}$ & $382.4 \pm 20.7^{\mathrm{e}}$ \\
Alkaloids $(\mathrm{mg} / \mathrm{mL})$ & $0.819 \pm 0.066^{\mathrm{a}}$ & $1.209 \pm 0.261^{\mathrm{b}}$ & $0.648 \pm 0.054^{\mathrm{c}}$ & $0.979 \pm 0.137^{\mathrm{d}}$ & $0.088 \pm 0.002^{\mathrm{e}}$ \\
Saponin $(\mathrm{mg} / \mathrm{mL})$ & $0.593 \pm 0.061^{\mathrm{a}}$ & $0.662 \pm 0.019^{\mathrm{a}}$ & $0.604 \pm 0.048^{\mathrm{a}}$ & $0.290 \pm 0.033^{\mathrm{b}}$ & $0.183 \pm 0.026^{\mathrm{c}}$ \\
\hline & & & &
\end{tabular}

ing it a good source of dietary niacin. The VB supply, namely the percentage ratio of VB in vinegar (100 g) relative to the recommended daily allowance of the same, was given in Fig. 4. As shown here, one hundred grams of vinegar can supply 22.3-455.4\% and 59.3$140 \%$ of the daily requirements of niacin and riboflavin daily for the human body. On average, a $35 \mathrm{~g}$ aliquot of OV would provide almost all an adult's daily niacin requirement $(25 \mathrm{mg})$, and $1 / 3$ of an adult's daily riboflavin requirement, as recommended by the Food and $\mathrm{Nu}$ trition Board, National Research Council ${ }^{12}$.

Nicotinic acid and nicotinamide are mainly present in plant-based and animal foods, respectively and abundant niacin was found in SAV in the present study. The raw materials (sorghum, oat, tartary buckwheat and particularly wheat bran) used to produce SAV are rich in niacin $^{33}$. For example, wheat bran has $12.5 \mathrm{mg} / 100 \mathrm{~g}$ of niacin, which is the highest among these raw materials. However, according to the change ratio of raw materials to vinegar products (generally, one kilogram of raw materials can produce three kilograms of vinegar), niacin in vinegar products will decrease to $1 / 3$ of its raw material quantity. Therefore, we speculated that microorganisms involved in vinegar making could synthesize this vitamin.

\section{Total phenolics, alkaloid and saponin}

Generally speaking, phenolics, alkaloids and saponin are considered major active fractions for curative effects in traditional Chinese medicine and food, hence the quantities contained in these vinegars were also determined.

The total phenolic contents decreased in the order of TBV (4518.1 $\mu \mathrm{g} / \mathrm{mL}), \mathrm{SV}(3964.7 \mu \mathrm{g} / \mathrm{mL}), \mathrm{OV}$

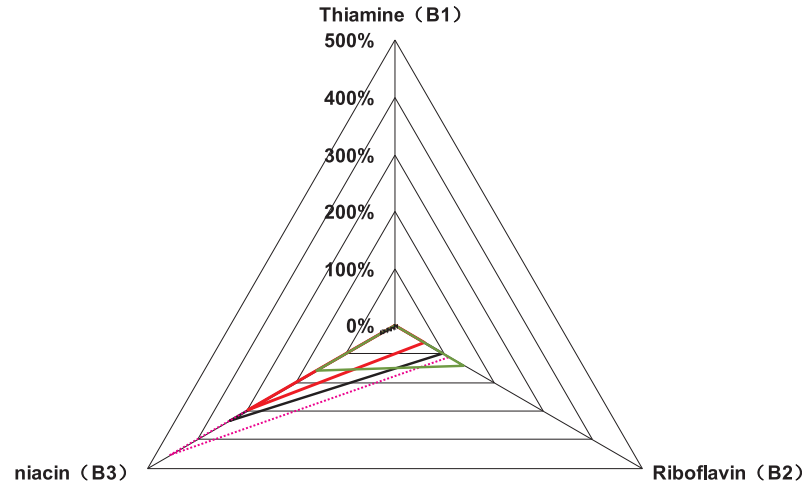

Fig. 4. Percentage ratio of three of the vitamin $B$ in vinegar relative to the recommended daily allowance

$$
\begin{array}{ll}
\cdots & : \mathrm{SV}, \cdots
\end{array}
$$

(2812.1 $\mu \mathrm{g} / \mathrm{mL})$, ZHV (1956.2 $\mu \mathrm{g} / \mathrm{mL})$ and LRV (382.4 $\mu \mathrm{g} / \mathrm{mL}$ ) (Table 6). SAV were significantly higher than ZAV in total phenolics $(p<0.05)$, which could be attributed to the high phenolic contents in minor grain crops $^{11}$.

In terms of total alkaloids (Table 6), a wide variation was observed. The OV was the highest $(1.209 \mathrm{mg} /$ $\mathrm{mL}$ ) in terms of total alkaloids, followed by $\mathrm{ZHV}$ (0.979 mg/mL), SV (0.819 mg/mL), TBV (0.648 mg/ $\mathrm{mL})$ and $\mathrm{LBV}(0.088 \mathrm{mg} / \mathrm{mL})$. Raw materials and microorganisms used in manufacturing could have a profound effect on the alkaloid content of these vinegars. The total contents of saponin in SAV had no significant differences $(p>0.05)$, although significantly exceeding that of ZAV. Generally speaking, the raw materials used for SAV production have a high saponin content ${ }^{33}$. 
Moreover, almost the same raw materials were used in different forms of SAV, such as SV and OV, which may explain why SAV have almost equivalent saponin content.

Compared with SAV and ZHV, LRV was low in minerals, Vitamin B complexes, phenols, alkaloids, and saponin (Tables 5, 6), which could be attributed to its raw materials, microorganisms in the koji and the aging period. Although LRV shares its manufacturing processes with ZHV, replacing the rice with starch, adding yeast to accelerate fermentation, a short aging period and high moisture content lowered its nutrients.

\section{7. $\alpha$-Glucosidase inhibitory activity}

It is well known that $\alpha$-glucosidase is an exoacting carbohydrase, the catalysis of which releases $\alpha$ D-glucopyranose from the non-reducing ends of various carbohydrate substrates ${ }^{13}$. These enzymes play an important role in the biochemical processes of glycoproteins and glycolipids ${ }^{4}$. The presence of $\alpha$-glucosidase inhibitor in diets can inhibit the activity of $\alpha$-glucosidase and reduce the absorption of dietary carbohydrates, meaning $\alpha$-glucosidase inhibitors might be useful in developing treatments for carbohydrate-mediated diseases such as diabetes, certain forms of hyperlipoproteinemia and obesity $^{3,8}$. It has been established that naturally occurring phenolics (catechin, Apigenin8-C-glucoside, anthocyanins, genistein, quercetin, chlorogenic acid), alkaloids (Berberine, 1,2-deoxynojirimycin) and saponin widely distributed in food have the ability to inhibit $\alpha$ glucosidase $^{6}$. To elucidate the influence of these bioac-

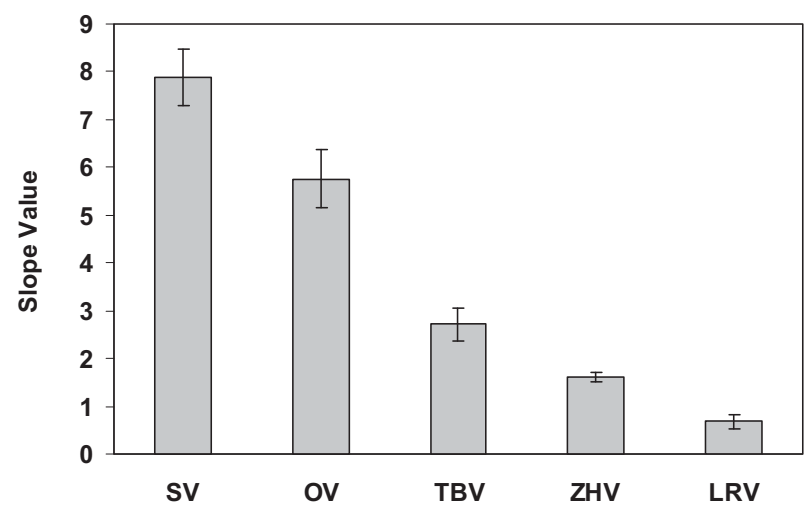

Fig. 5. The $\alpha$-glucosidase inhibitory activity of vinegar

Values represent the mean \pm standard deviation (SD) of $\mathrm{n}=3$ duplicate assays. SV, OV, TBV, ZHV and LRV represent sorghum vinegar, oat vinegar, tartary buckwheat vinegar, Zhenjiang Hengshun vinegar and Liubiju rice vinegar, respectively. tive substances on $\alpha$-glucosidase activity, the vinegars were neutralized and their $\alpha$-glucosidase inhibitory activity subsequently compared (Fig. 5). The results showed that although all the vinegars exhibited $\alpha$ glucosidase inhibitory activity, significant variations in the activity were found among them. The $\alpha$-glucosidase inhibitory activity of these vinegars followed the order: SV (7.9), OV (5.8), TBV (2.7), ZHV (1.6), LRV (0.7). On the whole, SAV were higher than ZAV in terms of $\alpha$-glucosidase inhibitory activity, which correlated to the higher contents of phenolics, alkaloids or saponins in the former. However, Pearson correlation analysis shows no relationship between phenolics, alkaloids or saponins and $\alpha$-glucosidase inhibitory activity among these SAV. For example, TBV had a higher total phenolic content, whereas its $\alpha$-glucosidase inhibitory activity was lower than SV, and OV (Fig. 5). Therefore, the $\alpha$-glucosidase inhibitory activity could result from the integrative effects of these substances, phenolics, alkaloids and saponin. Moreover, different types of phenolics, alkaloids and saponin could have different $\alpha$-glucosidase inhibitory activity, dependent on structure ${ }^{17}$. SV had been found to be rich in many phenolic acids, including gallic, 3,4-dihydroxybenzoic, chlorogenic, caffeic, vanillic, syringic and $p$-coumaric acids ${ }^{11}$, which could explain the high activity of SV. However, data concerning alkaloids and saponins in SV and phenolics, alkaloids and saponins in OV and TBV are scarce. These vinegars possibly contain different types of phenolics, alkaloids and saponin with different $\alpha$-glucosidase inhibitory activity. Further study should be conducted to elucidate the relationship between the $\alpha$-glucosidase inhibitory activity and the structure of the bioactive substances. The effect of raw materials and manufacturing processing, such as fermentation and roasting, on the changes in certain substances in vinegar are being studied in our laboratory.

\section{Conclusions}

This is the first nutrient report for the two most typical Chinese vinegars, the nutritional composition of which shows some significant differences. SAV, especially OV, is more abundant than ZAV in nutrients owing to its special raw materials and manufacture processing, including organic acids, amino acids, calcium, phosphorus, iron, zinc, manganese, niacin, phenolics, alkaloids and saponin. Its abundance in nutrients may open up special applications, including supplementing minerals for some women deficient in calcium and iron, supplying niacin to some people with niacin deficiency and extracting organic acids and amino acid dressings. 
The vinegars, especially SAV, were also found to have strong $\alpha$-glucosidase inhibitory activity, which may be useful for diabetics. However, the stimulus of the sour taste of vinegar may prove a major obstacle to efforts to promote its consumption in large quantities. In summary, Chinese vinegar may be exploited as part of the development of various functional foods for these attributes.

\section{Acknowledgments}

This study was supported by BLYX200920, BLX2 W8007, and 2008-BL-LY-01, Beijing Forestry University, China. This study was also supported by funds from the National Natural Science Foundation (grant no. 31071524).

\section{References}

1. Amerine, M. A. et al. (1967) The technology of wine making. The AVI Publishing Company Inc., Westport, Connecticut, USA.

2. Association of Official Analytical Chemistry (AOAC) (2000) Official Methods of Analysis of Analysis of the Association of Official Analytical Chemists, 17th ed. Washington, DC.

3. Baron, A. D. (1998) Postprandial hyperglycemia and $\alpha$-glucosidase inhibitors. Diabetes Res. and Cli. Pract., 40 (Suppl.), 51-55.

4. Bertozzi, C. R. et al. (2001) Chemical glycobiology. Science, 291, 2357-2364.

5. Carlavilla, D. et al. (2006) Chiral MEKC-LIF of amino acids in foods: analysis of vinegars. Electrophor., 27, 2551-2557.

6. Chen, J. et al. (2007) Research progress review on $\alpha$ glucosidase inhibitor in food. Food Sci., 28, 360-363 (In Chinese).

7. Cheng, B. Q. et al. (1987) Comparative effects of dietary wheat bran and its morphological components (aleurone and pericarp-seed coat) on volatile fatty acid concentration in the rat. Brit. J. Nutr., 57, 69-76.

8. de Melo, E. B. et al. (2006) $\alpha$ - and $\beta$-glucosidase inhibitors: Chemical structure and biological activity. Tetrahedron, 62, 10277-10302.

9. Dubois, M. et al. (1956) Colorimetric method for determination of sugars and related substances. Anal. Chem., 28, 350-356.

10. Ebihara, K. et al. (1998) Effect of acetic acid and vinegar on blood glucose and insulin responses to orally administered sucrose and starch. Agric. Biol. Chem., 52, 1311-1312.

11. Fan, J. F. et al. (2009) Antithrombotic and fibrinolytic activities of methanolic extract of aged sorghum vinegar. J. Agric. Food Chem., 57, 8683-8687.

12. Food and Nutrition Board, National Research Council (1989) Recommended dietary allowances, 10th ed. National Academy Press, Washington, DC, USA.

13. Frandsen, T. P. et al. (1998) Plant $\alpha$-glucosidases of the glycoside hydrolase family 31. Molecular properties, substrate specificity, reaction mechanism, and comparison with family members of different origin. Plant Mol. Biol., 37, 1-13.

14. He, Y. M. et al. (2008) Studies on preparative technology and quantitative determination for extracts of total saponin in roof of Panax japonicus. Zhongguo zhong yao Za Zhi, 33, 2607-2611 (In Chinese).

15. Horiuchi, J. I. et al. (1999) New vinegar production from onions. J. Biosci. Bioeng., 88, 107-109.

16. Huang, W. K. (1989) Food testing and analysis. Light Industry Press, Beijing, China.

17. Khan, A. R. et al. (2004) Synthesis of alphamannosylated phenolics as alpha-glucosidase inhibitors. J. Enzyme Inhib. Med. Chem., 19(2), 107-112.

18. Kishi, M. et al. (1999) Enhancing effect of dietary vinegar on the intestinal absorption of calcium in ovariectomized rats. Biosci. Biotechnol. Biochem., 163, 905-910.

19. Kondo, S. et al. (2001) Antihypertensive effects of acetic acid and vinegar on spontaneously hypertensive rats. Biosci. Biotechnol. Biochem., 65, 2690-2694.

20. Liu, D. et al. (2004) Chinese vinegar and its solid-state fermentation process. Food Rev. Int., 20, 407-424.

21. Long, S. J. et al. (2007) Determination of total alkaloid in zanthoxylum nitidum (Roxb.)DC. by bromophenol blue colorimetry. Food Sci., 28, 443-445 (In Chinese).

22. Ogawa, N. et al. (2000) Acetic acid suppresses the increasing in disaccharidase activity that occurs during culture of Caco-2 cells. J. Nutr., 130, 507-513.

23. Osborne, C. G. et al. (1996) Evidence for the relationship of calcium to blood pressure. Nutr. Rev., 54, 365381.

24. Peterson, D. M. et al. (2001) Phenolic antioxidants and antioxidant activity in pearling fractions of oat groats. J. Cereal Sci., 33, 97-103.

25. Satoshi, U. et al. (2003) Effect of vinegar on absorption of iron in rats. J. Jpn. Soc. Nutr. Food Sci., 56, 371-374.

26. Shimoji, Y. et al. (2002) Isolation and identification of DPPH radical scavenging compounds in Kurosu (Japanese unpolished rice vinegar). J. Agric. Food Chem., 50, 6501-6503

27. Svanberg, U. et al. (1997) Fermentation and nutrient availability. Food Control, 8, 319-327.

28. Wang, H. Y. et al. (2006) Nutritional value of sorghum and development and exploitation of resource. Food Res. Dev., 27, 91-93 (In Chinese).

29. White, A. M. et al. (2007) Vinegar ingestion at bedtime moderates waking glucose concentrations in adults with well- controlled type 2 diabetes. Diabetes Care, 30, 2814-2815.

30. Xu, Q. P. et al. (2003) Progress in vinegar function study. China Condiment, 12, 11-12 (In Chinese).

31. Yamagishi, K. et al. (1998) Purification and identification of blood fluidity improvement factor in brewed rice vinegar (Kurosu). Nippon shokushi kagaku kogaku kaishi, 45, 545-549 (In Japanese).

32. Yamaki, K. et al. (2006) Evaluation of alphaglucosidase inhibitory activity in Colored Foods: A trial using slope factors of regression curves. Nippon sho- 
kuhin kagaku kogaku kaishi, 53, 229-231 (In Japanese).

33. Yang, Y. X. (2004) China Food Composition. Peking University Medical Press, Beijing, China.

34. Ye, X. J. et al. (2004) In vitro evaluation of physiological activity of the vinegar produced from barley-, sweet potato-, and rice-shochu post-distillation slurry. Biosci.
Biotechnol. Biochem., 68, 551-556.

35. Yonemoto, C. et al. (1995) Effect of vinegar made from soybean oligosaccharides on lipid metabolism in rat. J. Jpn. Soc. Nutr. Food Sci., 48, 441-449.

36. Zemel, M. B. (1997) Dietary pattern and hypertension: the DASH study. Nutr. Rev., 55, 303-305. 